\title{
Eficácia e baixo custo no tratamento do ceratocone com o uso de lentes de contato rígidas gás-permeáveis
}

\author{
Efficacy and low cost in keratoconus treatment with rigid gas-permeablecontact lens
}

\author{
Tarciso Schirmbeck ${ }^{1}$ \\ Jayter S. Paula ${ }^{2}$ \\ Luiz Fernando Taranta Martin ${ }^{3}$ \\ Henrique Crósio Filho ${ }^{4}$ \\ Erasmo Romão 5
}

Trabalho realizado no Ambulatório de Oftalmologia do Hospital das Clínicas da Faculdade de Medicina de Ribeirão Preto da Universidade de São Paulo - USP.

Pós-graduando, nível doutorado, do Departamento de Oftalmologia, Otorrinolaringologia e Cirurgia de $\mathrm{Ca}$ beça e Pescoco da Faculdade de Medicina da Universidade de São Paulo - USP - Campus Ribeirão Preto (SP).

${ }^{2}$ Professor Colaborador do Departamento de Oftalmologia, Otorrinolaringologia e Cirurgia de Cabeça e Pescoço da Faculdade de Medicina da USP - Campus Ribeirão Preto (SP).

${ }^{3}$ Médico residente do Departamento de Oftalmologia, Otorrinolaringologia e Cirurgia de Cabeça e Pescoço da Faculdade de Medicina da USP - Campus Ribeirão Preto (SP).

${ }^{4}$ Médico Colaborador do Ambulatório de Lente de Contato do Departamento de Oftalmologia, Otorrinolaringologia e Cirurgia de Cabeça e Pescoço da Faculdade de Medicina da USP - Campus Ribeirão Preto (SP).

${ }_{5}^{5}$ Professor Assistente do Departamento de Oftalmologia, Otorrinolaringologia e Cirurgia de Cabeça e Pescoço da Faculdade de Medicina da USP - Campus Ribeirão Preto (SP).

Endereço para correspondência: Jayter Silva de Paula Departamento de Oftalmologia, Otorrinolaringologia e Cirurgia de Cabeça e Pescoço - Av. Bandeirante, 3900 $12^{\circ}$ andar - Ribeirão Preto (SP) CEP 14049-900

E-mail: jayterdepaula@msn.com

Recebido para publicação em 30.04.2004

Versão revisada recebida em 25.10.2004

Aprovação em 15.12.2004

Nota Editorial: Após concluída a análise do artigo sob sigilo editorial e com a anuência da Dra. Saly Bugman Moreira sobre a divulgação de seu nome como revisora dele, agradecemos sua participação nesse processo.

\begin{tabular}{l} 
RESUMO \\
\hline Objetivo: Avaliar a eficácia da adaptação de lente de contato rígida gás- \\
permeável (LCRGP) em pacientes com ceratocone es custos do tratamen- \\
to. Métodos: Realizou-se análise retrospectiva dos prontuários de 126 \\
pacientes atendidos no Ambulatório de Lente de Contato do Hospital das \\
Clínicas da Faculdade de Medicina de Ribeirão Preto no período de janeiro \\
de 2001 a junho de 2002. Foram analisados sexo, idade e número de olhos \\
adaptados. A acuidade visual sem e com correção foi relacionada à curva- \\
base em K (meridiano mais plano da córnea) do paciente e à diferença \\
ceratométrica dos meridianos corneanos. Determinou-se o custo unitário \\
médio das lentes fornecidas aos pacientes. Resultados: Obteve-se suces- \\
so na adaptação de lente de contato rígida gás-permeável em $86,19 \%$ dos \\
casos, com visão=0,8 em 40,00\% dos pacientes adaptados. Houve melhora \\
visual tanto nos pacientes com curva-base elevada quanto naqueles que \\
apresentavam alta diferença ceratométrica entre os meridianos corneanos. \\
A faixa etária mais acometida encontrou-se entre 15 e 45 anos, sendo \\
$60,31 \%$ dos pacientes do sexo feminino. A proporção de indicação de \\
transplante de córnea foi de $11,42 \%$. O custo unitário médio da lente foi de \\
R\$ 40,00. Conclusão: A adaptação proporcionou acentuada melhora \\
visual na maioria dos pacientes portadores de ceratocone a custo relati- \\
vamente baixo.
\end{tabular}

Descritores: Ceratocone/terapia; Lente de contato/economia; Topografia da córnea; Córnea/patologia; Adaptação fisiológica; Acuidade visual

\section{INTRODUÇ̃̃̃O}

O ceratocone é uma distrofia ectásica da córnea ${ }^{(1)}$ caracterizada por afilamento e alteração de sua curvatura que assume forma cônica, produzindo astigmatismos irregulares ${ }^{(2)}$. A incidência da doença varia de 20 a 230 por 100.000 habitantes ${ }^{(1-2)}$.

Essa afecção pode ser congênita ${ }^{(1)}$ mas geralmente se inicia na segunda década de vida ${ }^{(2)}$, progredindo durante 6 a $8 \operatorname{anos}^{(1-2)}$. Caracteriza-se por acometimento bilateral, freqüentemente de forma assimétrica ${ }^{(3)}$.

A etiologia do ceratocone é desconhecida. Várias possibilidades etiológicas foram estudadas e aventadas: influência genética ${ }^{(4)}$, alergia $^{(5)}$, uso de lentes de contato $^{(1)}$ e doenças do colágeno ${ }^{(2)}$. Estudos histopatológicos revelaram fragmentação da membrana de Bowman e da membrana basal do epitélio, afilamento epitelial e estromal, cicatrizes estromais e roturas da membrana de Descemet $^{(1-2)}$.

O principal sintoma dos pacientes portadores de ceratocone é a baixa 
acuidade visual (AV). O tratamento clínico do ceratocone inicia-se pelo uso de óculos. Com a progressão da doença, a adaptação de lentes de contato rígidas pode proporcionar boa visão.

O objetivo do presente estudo foi investigar a eficácia da adaptação de lentes de contato rígidas gás-permeáveis (LCRGP) em portadores de ceratocone e avaliar seu custo.

\section{MÉTODOS}

Foi realizado um estudo retrospectivo dos prontuários de 126 pacientes atendidos no Ambulatório de Lente de Contato do Hospital das Clínicas da Faculdade de Medicina de Ribeirão Preto (HCFMRP) no período de janeiro de 2001 a junho de 2002. Nesse ambulatório são adaptadas LCRGP exclusivamente em portadores de ceratocone. Foram excluídos do estudo os prontuários de pacientes com quaisquer outras afecções oculares e aqueles submetidos a procedimentos cirúrgicos oculares prévios.

O método de adaptação utilizado no ambulatório é o da tentativa e erro ${ }^{(1)}$, no qual se inicia a adaptação com a lente de prova de curvatura posterior igual à curva-base $(\mathrm{K})$ do paciente, seguido de aumento da curvatura ou diâmetro da lente de prova conforme a adaptação obtida.

O ceratocone foi classificado conforme sua curvatura mais plana em dioptrias (D) como: incipiente ( $<45 \mathrm{D})$, estabelecido (45-51,99 D), avançado (52- 60 D) e muito avançado (>60 D).

Foram coletados dados sobre sexo, idade, número de olhos adaptados e AV. Na avaliação da visão levaram-se em conta os valores da AV sem correção, com óculos e com LCRGP. Relacionou-se a AV à curva-base da córnea do paciente e à diferença ceratométrica entre as curvaturas corneanas. Para lentes de contato do tipo lenticular e do tipo Soper foram coletadas também informações sobre o valor ceratométrico da curva posterior da lente adaptada em relação à curvabase da córnea do paciente.

Os casos nos quais houve melhora satisfatória da acuidade visual, boa tolerabilidade e adequada qualidade de adaptação com o uso de LCRGP, foram considerados como de sucesso e expressos em porcentagem.

A média do custo unitário de cada LCRGP foi obtida através do cálculo dos valores constantes nos recibos de pagamento arquivados da instituição.

\section{RESULTADOS}

A adaptação de LCGRP foi realizada em 210 olhos dos 126 pacientes avaliados; destes, 76 eram do sexo feminino $(60,31 \%)$ e 50 do masculino $(39,69 \%)$. A adaptação foi realizada em um olho em 42 pacientes e nos dois olhos em 84; sendo 106 no direito e 104 no esquerdo. A faixa etária mais acometida encontrou-se entre 15 e 45 anos (Tabela 1).

Foi encontrada maior proporção de pacientes $(43,33 \%)$ com ceratocone estabelecido (Tabela 2). Observou-se AV baixa $(=0,2)$ sem qualquer correção em $76,78 \%$ dos casos, AV razoável com uso de óculos $(66,88 \%$ entre 0,1 e 0,5$)$ e AV satisfatória quando se fez a correção com LCRGP (72,18\% dos pacientes com $\mathrm{AV}=0,5$ e 40,00\% com $\mathrm{AV}=0,8$ ) (Figura 1).

Verificou-se queda gradativa da AV sem correção com o aumento da curva-base: $29,71 \%$ dos pacientes com ceratocone do grau incipiente apresentavam AV entre 0,5 e 1,0; 48,80\% do grau estabelecido tinham AV entre 0,2 e 0,5 e $80 \%$ do grau avançado apresentavam $\mathrm{AV}<0,2$. Outro fator que influenciou a visão foi a diferença ceratométrica dos meridianos mais curvo e mais plano da córnea (Figura 2).

Correlacionou-se a curva-base $(\mathrm{K})$ do olho à curvatura posterior da lente melhor adaptada. Observou-se que nas lentes do tipo lenticular o valor da curva posterior da lente adaptada situou-se entre 0 e 2,5 D acima de $\mathrm{K}$ na maioria dos pacientes (66\% dos pacientes com curva-base menor que $45 \mathrm{D}$ ou entre 45 e 51,99 D; figura 3). Nas outras curvas-base e com as lentes tipo Soper não se observou nenhum padrão específico para adaptação.

Constatou-se que a maioria dos pacientes $(86,19 \%)$ teve sucesso com lentes de contato. A indicação de transplante de córnea foi de $11,42 \%$ dos olhos estudados, tendo estes em sua quase totalidade $\mathrm{K}$ acima de $52 \mathrm{D}$ (Tabela 2).

O custo unitário médio de cada LCRGP foi de R \$40,00.

\section{DISCUSSÃO}

O ceratocone constitui uma afecção que proporciona acuidade visual insatisfatória aos pacientes acometidos. Tal fato se deve às alterações anatômicas presentes na córnea ${ }^{(1)}$, na qual se observa uma importante ectasia central ou paracentral com distorção da curvatura corneana ${ }^{(2)}$, resultando em astigmatismos bastante irregulares ${ }^{(6)}$. Grande parte dos pacientes com ceratocone obtém boa visão somente com LCRGP, fato confirmado no presente trabalho.

A acuidade visual sem correção está relacionada à curva-

\begin{tabular}{|c|c|}
\hline Idade (anos) & Número de pacientes \\
\hline $1-5$ & 0 \\
\hline $6-10$ & 0 \\
\hline $11-15$ & 10 \\
\hline $16-20$ & 31 \\
\hline $21-25$ & 24 \\
\hline $26-30$ & 21 \\
\hline $31-35$ & 17 \\
\hline $36-40$ & 11 \\
\hline $41-45$ & 3 \\
\hline $46-50$ & 4 \\
\hline Maior de 50 & 5 \\
\hline Total & 126 \\
\hline
\end{tabular}




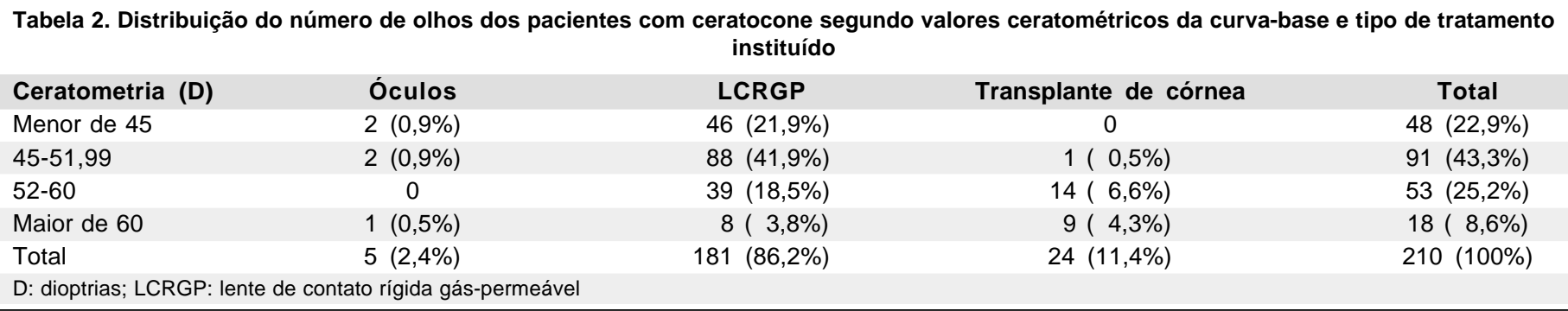

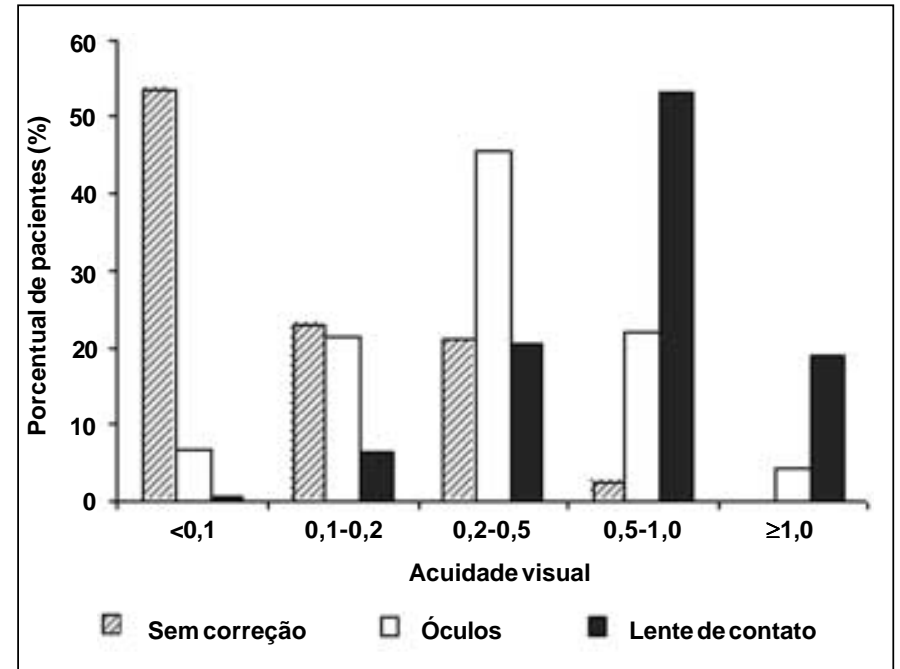

Figura 1 - Correlação entre porcentual de pacientes com ceratocone sem correção $(n=173)$, submetidos à correção com óculos $(n=145)$, LCRGP ( $n=169)$ e acuidade visual alcançada

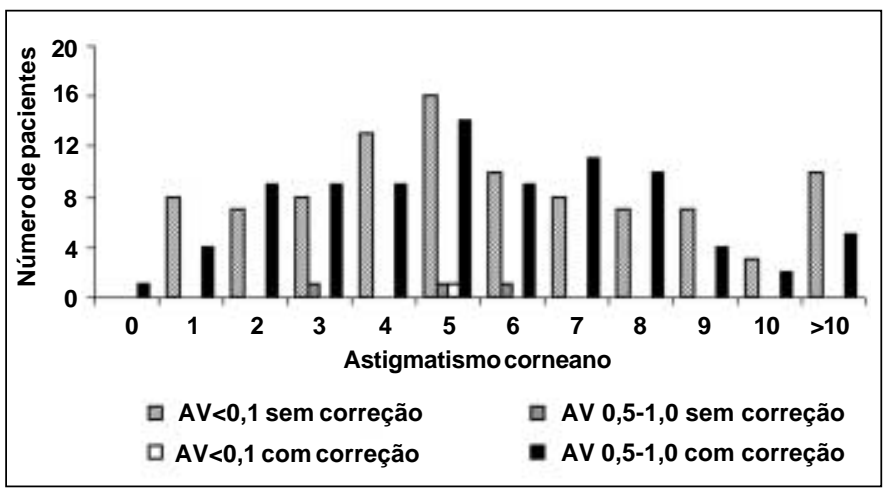

Figura 2 - Correlação entre número de pacientes com ceratocone, acuidade visual alcançada (com e sem correção) e astigmatismo corneano (em dioptrias)

tura mais plana da córnea e à diferença ceratométrica entre o meridiano mais plano e o mais curvo. Valores $\mathrm{K}$ mais planos, em torno de $45 \mathrm{D}$, possibilitam visão razoável, enquanto que valores além de $60 \mathrm{D}$ resultam em visão pior que 0,1 , conforme observado em $91,66 \%$ dos nossos pacientes. Do mesmo modo, alguns trabalhos confirmam queda da visão quando há diferença ceratométrica maior que $6,0 \mathrm{D}^{(7)}$.

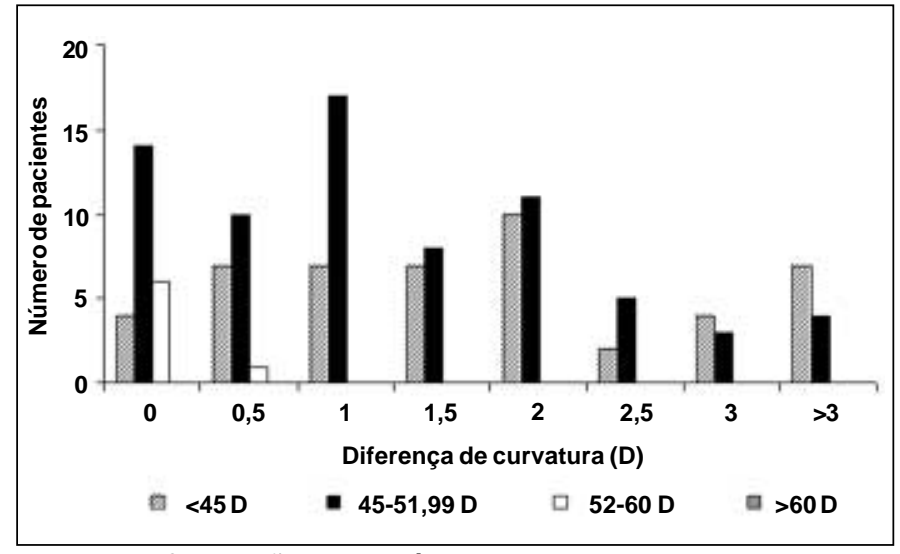

Figura 3 - Correlação entre número de pacientes com ceratocone incipiente (K<45D), estabelecido (K 45-51,99D), avançado (K 52-60D) e muito avançado (K>60D), e diferença de curvatura, em dioptrias (D), entre a curva-base de cada paciente $(K)$ e a curva posterior da lente adaptada (tipo lenticular)

Alguns casos de ceratocone incipiente apresentam correção razoável da visão com óculos. Em casos mais avançados, somente as LCRGP podem proporcionar boa visão. O uso das LCRGP promove regularização da curvatura corneana, corrigindo as aberrações sofridas pelos raios luminosos ${ }^{(6-7)}$. Essa correção ocorre tanto em curvas-base elevadas, quanto em grandes diferenças ceratométricas (Figura 2). Vários trabalhos demonstram a excepcional melhora da visão obtida com LCRGP, alguns com $66,67 \%^{(8)}$, outros com $90,5 \%{ }^{(7)}$ dos pacientes com visão $=0,5$, valores próximos aos observados no presente estudo.

Existem vários métodos descritos para se adaptar lentes de contato em pacientes com ceratocone, alguns com uso de aparelhagem sofisticada, como topografia ${ }^{(9)}$ e videoceratoscopia $^{(1)}$, muitas vezes não disponíveis a todos os oftalmologistas. Todavia, todas as técnicas utilizadas têm apresentado resultados comparáveis no sucesso da melhora da visão dos pacientes ${ }^{(1,9)}$. Certos trabalhos buscam estabelecer protocolos de adaptação que visem facilitar a escolha da lente mais adequada; alguns através da adaptação da LCRGP mais plana que $\mathrm{K}^{(10)}$, outros pelo valor médio de $\mathrm{K} 1,5 \mathrm{~mm}$ acima a $90^{\circ}$ na topografia de córnea ${ }^{(9)}$, outros com livramento apical ${ }^{(11)}$. Nosso trabalho revelou certa regularidade de adaptação somente nos pacientes com $\mathrm{K}$ menor do que 51,99 $\mathrm{D}$ e adaptados com lentes do tipo lenticular (Figura 3). Nos demais casos, prova- 
velmente devido à intensidade das irregularidades corneanas, cada paciente teve de ser avaliado individualmente de acordo com suas características.

A decisão final sobre o tipo de tratamento a ser instituído obedeceu à análise da melhor acuidade visual atingida, à qualidade da adaptação avaliada pelo oftalmologista, ao grau de tolerabilidade à LCRGP pelo paciente e aos critérios médicos de indicação de lentes de contato. A proporção de $86,19 \%$ de sucesso e $11,42 \%$ de indicação de transplante de córnea concorda com dados da literatura que relatam sucesso na adaptação em $84,68 \%{ }^{(12)}$, proporção de indicação de transplante de 10 a $20 \%{ }^{(1)}$ e comprovam a eficácia na adaptação de LCRGP no ceratocone.

Destaca-se a contribuição do Ambulatório de Lente de Contato do HCFMRP aos pacientes, principalmente considerando-se o baixo nível sócioeconômico da população atendida. Constatou-se que $92,85 \%$ dos pacientes com ceratocone estão situados na faixa etária entre 15 e 45 anos, aquela considerada economicamente ativa. Tal achado também é mencionado na literatura ${ }^{(1,6)}$.

Segundo o IBGE ${ }^{(13)}$, a renda mensal do brasileiro nesta faixa etária é de aproximadamente dois salários-mínimos (em torno de $\mathrm{R} \$ 480,00$ naquele período). O Ambulatório de Lentes de Contato do HCFMRP considera terapêutica e não estética a indicação de LCRGP nesses casos. Através de programa especial promove o fornecimento gratuito da lente aos pacientes. $\mathrm{O}$ custo médio de cada lente ao HCFMRP foi de R \$ 40,00. Estimando-se o uso de um par de lentes por paciente e sua durabilidade em um ano, o custo anual para melhorar a visão de cada paciente portador de ceratocone seria de $\mathrm{R} \$ 80,00$.

Ressalte-se que grande parcela dos pacientes da lista de espera para transplante de córnea é constituída por portadores de ceratocone que não tiveram a oportunidade de passar por teste de adaptação de lentes de contato.

\section{CONCLUSÃO}

Cumpre indagarmos se não seria desejável e possível ao sistema público de saúde promover o tratamento adequado à demanda reprimida de pacientes portadores de ceratocone sem acesso ao serviço particular.

\section{ABSTRACT}

Purpose: To study the efficacy of rigid gas-permeable contact lens (RGPCL) fitting in keratoconus patients and to determine treatment costs. Methods: The records of 126 patients treated at the Hospital das Clínicas de Ribeirão Preto from January 2001 to
June 2002 were retrospectively studied. Information about sex, age and treated eyes was collected. Visual acuity (VA) with and without correction was measured and plotted against corneal base curve $(\mathrm{K})$ and toricity. Average price of the lenses was calculated. Results: Fitting rigid gas-permeable contact lens was successful in $86.18 \%$ of the patients, resulting in $\mathrm{VA}=0.8$ in $40.00 \%$ of them. Visual acuity improvement was observed in patients with steep base curve and in patients with high corneal toricity. Age of most patients ranged from 15 to 45 years; $60.31 \%$ of them were female. Corneal transplantation was indicated in $11.24 \%$ of the cases. Cost of each lens averaged $\mathrm{R} \$ 40.00$ (US\$ 13.00). Conclusion: Rigid gas-permeable contact lens proved to be an efficient, low-cost resource for improving visual acuity in most of the treated patients.

Keywords: Keratoconus/therapy; Corneal topography; Contact lens/economics; Cornea/pathology; Adaptation, physiological; Visual acuity

\section{REFERÊNCIAS}

1. Felder RS. Noninflammatory ectatic disorders. In: Krachmer JH, Mannis MJ, Holland EJ. editors. Cornea, diagnosis and management. St. Louis, Missouri: Mosby Year Book; 1997. p.1091-100.

2. Belfort Jr R. Ceratocone. In: Belfort Jr R, Kara-José N. editores. Córnea clínica e cirúrgica. São Paulo: Roca; 1997. p.359-65.

3. Zadnik K, Barr JT, Edrignton TB, Everett DF, Jameson M, McMahon TT, et al. Baseline findings in the Collaborative Longitudinal Evaluation of Keratoconus (CLEK) study. Invest Ophthalmol Vis Sci. 1998;39(13):2537-45.

4. Urbano AP, Urbano AP, Urbano I, Jose NK. Influência genética no ceratocone. Rev Bras Oftalmol. 2002;61(2):73-6.

5. Totan Y, Hpsen IF, Çekiç O, Gündüz A, Aydin E. Incidence of keratoconus in subjects with vernal keratoconjuntivitis: a video keratographic study. Ophthalmology. 2001;108(4):824-7.

6. Cunha MC, Lima ALH de. Ceratocone. Estudo comparativo entre pacientes adaptados com lentes de contato e pacientes com indicação de transplante de córnea. Rev Bras Oftalmol. 1997;56(7):495-511.

7. Leite Neto AQ, Florêncio ACT, Barroca RP, Sales C, Lucena AR. Avaliação do uso de lentes de contato no ceratocone. Rev Bras Oftalmol. 2001;60(5):364-8.

8. Passos MC, Pereira MLM, Zauberman TA, Monica LAM. Ceratocone; estudo retrospectivo de 103 olhos. Rev Bras Oftalmol. 2000;59(4):255-61.

9. Gomes JAP, Lani LA, Juliano Y, Gomes R, Pedro EA, Anbar R. Uso da topografia de córnea na adaptação de lentes de contato rígidas gás-permeáveis em pacientes portadores de ceratocone: descrição de técnica e resultados preliminares. Arq Bras Oftalmol. 2002;65(5):519-23.

10. Edrington TB, Szczotka LB, Barr JT, Achtenberg JF, Burger DS, Jannof AM et al. Rigid contact lens fitting relationship in keratoconus. Optom Vis Sci. 1999;76(10):692-9. Comment in: Optom Vis Sci. 2000;779(4):177.

11. Gundel RE, Libassi DP, Zadnik K, Barr JT, Davis L, McMahon TT et al. Feasibility of fitting contact lens with apical clearance in keratoconus. Optom Vis Sci. 1996;73(12):729-32.

12. Ghanem VC, Ghanem CC, Gahnem RC, Larinho C. Ceratocone: correlação entre o grau evolutivo e padrão topográfico com o tipo de lente de contato adaptada. Arq Bras Oftalmol. 2003;66(2):129-35.

13. Instituto Brasileiro de Geografia e Estatística. Departamento de Contas Nacionais do IBGE. Rendimento médio mensal - Brasil. Trabalho e Rendimento 1999 [texto na Internet]. Disponível em: http://www.ibge.gov.br/home/estatística/ população/condiçãodevida/indicadoresminimos/tabela2.shtm\#a111. 